\title{
Harnessing and engineering amide bond forming ligases for the synthesis of amides
}

DOI:

10.1016/j.cbpa.2019.12.004

10.1016/j.cbpa.2019.12.004

\section{Document Version}

Accepted author manuscript

Link to publication record in Manchester Research Explorer

\section{Citation for published version (APA):}

Winn, M., Richardson, S., Campopiano, D. J., \& Micklefield, J. (2020). Harnessing and engineering amide bond forming ligases for the synthesis of amides. Current Opinion in Chemical Biology, 55, 77-85.

https://doi.org/10.1016/j.cbpa.2019.12.004, https://doi.org/10.1016/j.cbpa.2019.12.004

\section{Published in:}

Current Opinion in Chemical Biology

\section{Citing this paper}

Please note that where the full-text provided on Manchester Research Explorer is the Author Accepted Manuscript or Proof version this may differ from the final Published version. If citing, it is advised that you check and use the publisher's definitive version.

\section{General rights}

Copyright and moral rights for the publications made accessible in the Research Explorer are retained by the authors and/or other copyright owners and it is a condition of accessing publications that users recognise and abide by the legal requirements associated with these rights.

\section{Takedown policy}

If you believe that this document breaches copyright please refer to the University of Manchester's Takedown Procedures [http://man.ac.uk/04Y6Bo] or contact uml.scholarlycommunications@manchester.ac.uk providing relevant details, so we can investigate your claim.

\section{OPEN ACCESS}




\title{
Harnessing and Engineering Amide Bond Forming Ligases \\ for the Synthesis of Amides
}

Michael Winn, ${ }^{a}$ Shona Richardson, ${ }^{b}$ Dominic J. Campopiano*,b and Jason Micklefield*,a

\begin{abstract}
aSchool of Chemistry and Manchester Institute of Biotechnology (MIB), The University of Manchester, 131 Princess Street, Manchester M1 7DN, UK.

bSchool of Chemistry, University of Edinburgh, David Brewster Road, King's Buildings, Edinburgh, EH9 3FJ, UK.
\end{abstract}

Correspondence:

Email: Dominic.Campopiano@ed.ac.uk; Tel: +44 (0)131 6504712

Email: jason.micklefield@manchester.ac.uk; Tel: +44 (0)161 3064509

\begin{abstract}
The amide functional group is ubiquitous in nature and one of the most important motifs in pharmaceuticals, agrochemicals and other valuable products. Whilst coupling amides and carboxylic acids is a trivial synthetic transformation, it often requires protective group manipulation, along with stoichiometric quantities of expensive and deleterious coupling reagents. Nature has evolved a range of enzymes to construct amide bonds the vast majority of which utilize adenosine triphosphate (ATP) to activate the carboxylic acid substrate for amine coupling. Despite the fact that these enzymes operate under mild conditions, as well as possessing chemoselectivity and regioselectivity that obviates the need for protecting groups, their synthetic potential has been largely unexplored. In this review we discuss recent research into the discovery, characterisation and development of amide bond forming enzymes, with an emphasis on stand-alone ligase enzymes that can generate amides directly from simple carboxylic acid and amine substrates.
\end{abstract}




\section{Introduction}

The coupling of carboxylic acids and amines to form amide bonds is one of the most fundamental reactions in chemistry and biology. Many top pharmaceuticals include one or more amide bonds, with acylation of amines accounting for $16 \%$ of all reactions performed by the pharmaceutical industry [1, 2]. Although the coupling of acids and amines is synthetically straightforward, it typically requires harmful reagents and solvents as well as stoichiometric quantities of costly coupling reagents which can cause problems with reaction purification. Moreover, in many cases atom inefficient protecting group chemistries are required, which means that multiple synthetic steps (protection-coupling-deprotection) are needed to introduce a single amide bond [3]. Consequently, there is an urgent need for new, more efficient, sustainable methods for the production of amides. In addition to proteins and peptide natural products, produced by ribosomes and non-ribosomal peptide synthetase (NRPS) enzymes, amides are present in a vast array of other primary and secondary metabolites. Nature has evolved a range of ligase enzymes catalysing amide bond formation that function via adenosine-5'-triphosphate (ATP)-dependent activation of a carboxylic acid substrate (Figure $1 \mathrm{~A}$ ). One notable exception to this general theme, is the ATP-independent CapW enzyme (Figure 1A, vi) that ligates a methyl ester and amine precursors in the biosynthesis of the antibiotic A-503083B (9). In this case an S-adenosylmethionine (SAM)-dependent methyltransferase CapS, methylates and activates the carboxylic acid precursor [4]. Given that such ATP-independent amide-forming ligases are rare, this review will focus on the more common ATPdependent ligases, particularly those ligases where ATP-activation is followed by direct attack of an amine nucleophile (Figure 1A, i). ATP-dependent acyl-activation, followed by attack of coenzyme A (CoA) to form an intermediate acyl-CoA thioester which is subsequently coupled with an amine by a second $N$-acyltransferase (NAT) enzyme, is also a common theme in amide biosynthesis (Figure $1 A$, ii). However, the requirement of two enzymes could limit potential applications of such systems for more general amide synthesis. While some NATs have been shown to be fairly promiscuous, many studied acyl-CoA synthetases are not and the substrate scope of both enzymes needs to be complimentary for this approach to be successful [5]. Despite these potential problems this review will also examine a few examples where high throughput screens have been used to identify combinations of enzymes that are effective and where enzyme engineering has broadened the substrate scope of acyl-CoA synthetases for the synthesis of specific, useful amide products [5-7]. Other enzymes including hydrolase enzymes such as lipases and penicillin acylases have been used in the synthesis of amides, however these enzymes have been extensively covered elsewhere and will not be discussed here [8-11]. 
The ATP-dependent ligase enzymes can be split into two broad families; adenylate forming enzymes that generate acyl-adenylate (acyl-AMP) intermediates eliminating pyrophosphate (PPi) (Figure 1A, i-iv) and ATP-grasp enzymes (Figure 1A, v), which form an acyl-phosphate intermediate with the release of adenosine diphosphate (ADP) (Figure $1 A$ ). The adenylate forming family of ligases can be further subdivided into four classes (Figure 1B). The first class, known as the ANL superfamily, includes Acyl/Aryl-CoA ligases (A), adenylation domains from nonribosomal peptide synthesis (N) and luciferase enzymes $(L)$. The second class are the aminoacyl-tRNA synthetases and the third are NRPS-independent siderophores. More recently, another subclass of ANL ligases with potential for biocatalysis, has been discovered, named the pimeloyl-CoA synthetases (PCAS), as defined by BioW $[12,13]$. This new enzyme exhibits no sequence homology to any other member of the ANL superfamily and indeed, displays a novel 3D protein fold. However structural and mechanistic analysis showed that the enzyme follows the same general adenylate-forming mechanism as described previously so merits definition as the newest member of the ANL family as class IV (Figure 1B) $[12,13]$. Aside from the more unusual pimeloyl-CoA synthetases, members of the ANL superfamily share a similar structure with a large $\mathrm{N}$-terminal domain and a smaller $\mathrm{C}$-terminal domain, the active site usually lies between these two subunits. Following the initial reaction to form the acyl-adenylate the C-terminal domain undergoes a large rotation $\left(140^{\circ}\right)$ which forms the binding pocket for the nucleophile (Figure 1B). In most cases this is coenzyme $A$, or in the case of NRPS the phosphopantetheine (4' -PP) arm of a PCP (derived from CoASH) which is subsequently processed by condensation enzyme to form the final amine product. A sub class of ANL enzymes also exists in which this binding pocket accepts an amine directly with no involvement of a separate acyl carrier protein, these are known as ATP-dependent amide bond synthetases (ABS) and they have been identified within several secondary metabolite clusters such as novobiocin (10) [14], clorobiocin (11) [15] and Xiamenmycin (12) [16].

\section{Applications of amide bond synthetases (ABS) and ATP-grasp enzymes in amide synthesis.}

The ABS family are potentially the most versatile enzymes for synthetic applications, as they are relatively simple, single (standalone) enzymes capable of directly coupling carboxylic acid and amine substrates. For example, ABSs NovL, CloL, and CouL from aminocoumarin biosynthetic pathways, producing novobiocin (10), clorobiocin (11) and coumermycin A (13) respectively (Figure 2A), were all screened with 22 synthetic carboxylic acid substrates that included benzoic acid derivatives with methyl, keto, amino, halide, alkyl, $\mathrm{N}$-alkyl and cyclohexyl substituents. While all three enzymes accepted various benzoate substrates, CloL was found to be the most promiscuous [17]. A related 
ABS enzyme XimA, from Streptomyces xiamenensis, which ligates the benzopyran precursor with Lthreonine in Xiamenmycin (12) biosynthesis, was also tested with 20 different amino acids, but ligation was only observed with the cognate (L-threonine) amino acid [16]. McbA, another ABS isolated from marine bacterium Marinactinospora thermotolerans, catalyses the condensation of a $\beta$-carboline (1-acetyl-3-carboxy- $\beta$-carboline) derivative with 2-phenyethylamine to form an amide (14) [18]. Notably McbA was shown to possess considerable substrate flexibility accepting a large range of benzoic acids, naphtoic acids, indole carboxylic acids, quinolines and benzufuran carboxylic acid substrates (Figure 2B) [19]. McbA also exhibits flexibility towards different amine substrates, with a large number of $\beta$-phenylethylamine and tryptamines successfully intercepting acyl adenylate intermediates forming a range of amide products (Figure 2B) [18]. Structural studies of a K483A mutant of McbA with AMP and $\beta$-carboline bound showed few specific active site interactions with the bound substrate, which was subsequently proposed as the reason for the observed substrate promiscuity $[18,19]$. This unusually broad substrate scope has highlighted McbA as one of the more promising candidate ABS for future biocatalytic amide synthesis.

The synthetic potential of ATP-grasp enzymes has also been explored. For example, a number of L-amino acid ligases (Lal) have been characterised which can couple L-amino acids to form dipeptides via an amino acyl phosphate intermediate, with TabS possessing the broadest substrate specificity described to date [20]. Another ATP-grasp enzyme, PolG catalyses amide bond formation between the unusual amino acid carbamoylpolyoxamic acid (CPOAA) and an amino nucleoside during the biosynthesis of the peptidyl nucleoside antibiotic polyoxin (15). Precursor feeding experiments showed that other nucleoside analogs could be accepted by this enzyme to produce modified polyoxins, suggesting flexible substrate scope [21]. More recently the in vitro activity of PolG has been demonstrated and shown to accept a diverse range of nucleoside substrates which may indicate significant potential for this class of amide forming enzymes for the synthesis of novel peptidyl nucleoside antibiotics [22].

ATP-grasp enzymes that accept peptides as substrates have also recently been identified. The first of these to be characterised is PGM1 from the biosynthetic gene cluster of the pheganomycins (16) (PGMs), a family of related compounds which consist of the nonproteinogenic (S)-2-(3,5-dihydroxy-4-hydroxymethyl)phenyl-2-guanidinoacetic acid ligated to the N-terminus of a ribosomally synthesised core peptide, which can range in length from 3 to 7 amino acids. Structural analysis of PGM1 revealed a long peptide binding cleft in the active site, which allows peptides of varying lengths and compositions to be accepted in addition to the natural substrates, including unrelated peptides such as aspartame (D-F methyl ester), a commercially available tetrapeptide 
(MRFA) and even the antibacterial peptide apidaecin (consisting of 18 amino acids). This indicates an extremely wide substrate scope, suggesting applications including peptide labelling and the engineering of novel bioactives [23]. A similar enzyme has also recently been implicated in the biosynthesis of the related compound resorcinomycin [24]. Subsequently a number of PGM1 orthologs have been identified from clusters, unrelated to either pheganomycin or resorcinomycin, from three different actinobacterial strains, leading to the discovery of a group of structurally related pseudotripeptides called ketomemicins, which all contain an amidino-arginine at the $\mathrm{N}$ terminus. One of these enzymes, from Micromonospora sp., was characterised and found to be functional as a novel dipeptide ligase, catalysing by amide bond formation between L/D-amidinoarginine, L/D -phenylglycine, 2-guanidinoacetic acid or creatine (2-(1-methylguanidino)acetic acid) and a range of dipeptides [25].

\section{Engineering amide bond forming enzymes.}

The limited substrate scope of some amide bond forming enzymes can be addressed through enzyme engineering. For example, significant attention has been focused on engineering nonribosomal peptide synthetase (NRPS) enzymes to produce novel peptide products. Early attempts to modify NRPS enzymes relied on active site mutagenesis of adenylation (A) domains, altering their selectivity for different amino acids substrates. Alternatively, individual A-domains or whole NRPS modules were exchanged to change the sequence of the peptide product. However, this met with limited success due to the importance of maintaining effective linker regions between adjacent modules [26]. More recently a flavodoxin-like sub domain was identified in the N-terminal domain of the phenylalanine activating A domain of GrsA which contains all of the important specificity encoding residues. The nature of this domain allowed it to be excised and replaced with the corresponding regions of a number of different A domains while maintaining the crucial NRPS interdomain interactions (Figure $3 \mathrm{~A}$ ). These changes showed a switch in substrate selection by in vitro assays [27], suggesting a new route to modified natural products by directly altering the substrate recognition of the adenylation domain. However, the subsequent downstream selectivity of condensation and thioesterase domains and hydrolytic proofreading functions of type II thioesterases still limit the incorporation of radically altered amino acids in the final structure.

Standalone A-domains excised from NRPS enzymes have also been successfully used in isolation for amide bond formation. For instance, a diverse range of tryptophyl- $N$-alkylamides have been produced by combining the tryptophan activating A domain from the first module of tyrocidine synthetase with a range of primary or secondary amines such as methylamine, piperidine, and cis- 
4-hydroxy-L-proline [28]. A 3-isocyanobutanoic acid activating A domain from the NRPS assembly line of SF2768 (17), a chalkophore isolated from Streptomyces thioluteus, has been shown to be promiscuous for 23 out of a potential library of 75 diverse carboxylic acids including; fatty acids, unsaturated fatty acids, dicarboxylic acids, halogenated acids, aromatic acids, and amino acids. The fatty, unsaturated fatty and halogenated acids were the preferred substrates for this enzyme. An enzyme cascade composed of this A domain together with three other enzymes from the pathway (including an NRPS, a standalone PCP and a hydroxylase) enabled 16 of these acid to be assembled into SF2768 analogs (Figure 3B) [29]. During the biosynthesis of tambjamine YP1, an adenylating domain present within the enzyme TamA activates a fatty acid substrate which is captured by the phosphopantetheine (4'-PP) prosthetic group before undergoing further processing steps to generate a long chain amine [30]. In isolation, this adenylation domain was shown to accept fatty acids ranging in length from $\mathrm{C} 6$ to $\mathrm{C} 14$ and resulting acyl adenylates could be captured by a range of amines, including both L- and D-amino acids, benzylamines and ethanolamine, to generate novel fatty acid amides (Figure 3C) [31]. In a similar fashion, carboxylic acid reductases (CARs) have been used to produce amides. These enzymes, possess an adenylation domain, carrier protein and reductase domains, which normally function to adenylate, thiolate and then reduce a carboxylic acid substrate to the corresponding aldehyde $[32,33]$. The reductase domains in a series of these CAR enzymes, from various microorganisms, were deactivated. The resulting mutants were shown to mediate amide bond formation with a range of amines and acids. The fact that up to a 100 fold excess of amine was required, suggests that the CAR mutants catalyse adenylation and thiolation, and that the resulting acyl-adenylate and/or thioester intermediates then undergo spontaneous (non-enzymatic) attack by the amine nucleophile [34]. Indeed, it is likely than none of the adenylating domains, used in isolation, as described here are capable of catalysing amide bond formation [28, 31, 34]. Notwithstanding this, the broad substrate scope of the CAR system, did enable synthesis of the amide anticonvulsant drug ilepcimide (18) in 96\% yield after an extended 3 day incubation (Figure 3D). To probe the mechanism of amide bond formation, the Ser698 residue of CARni was mutated to alanine, preventing post-translation modification with the 4'-PP group and subsequent thiolation, leading to accumulation of the acyl adenylate. In this case the ilepcimide (18) was formed in slightly reduced $79 \%$ yield, suggesting attack of acyl adenylate by the amine is the main reaction pathway. Also, a truncated forms of CARmm (with the reductase domain removed altogether) were also able to produce ilepcimide (18) in 69\% yield.

The acetyl-CoA synthetase from Arabidopsis thaliana (atACS) has also recently been engineered to broaden its substrate selectivity. This enzyme catalyses adenylation of acetate to 
generate acetyl-AMP and subsequent thiolation with coenzyme A. There are a large number of similar acyl-CoA synthetase enzymes in nature, but these enzymes usually exhibit narrow substrate selectivity. Multiple sequence alignments and structural comparisons of acyl-CoA synthetases identified four residues (1323, T324, V399 and W427) that seemed to be key for substrate recognition. These residues in atACS were mutated to the corresponding residues found in the isobutyrate selective CoA-synthetase from Pseudomonas chlororaphis (pclCS) (making mutations I323A, T324Y, V399A, W427G). Two single site mutations (W427G and V399A) resulted in altered carboxylic acid recognition. The W427G mutation switching preference from acetate to longer, straight chain substrates ranging from 3 to 8 carbons in length, with a corresponding 6.5-fold decrease in activity for the natively recognised acetate. The V399A mutant allowed the enzyme to retain acetate activity while also accepting propionate on an equal basis. The double mutation exacerbated the effects with an 80-fold increase in activity with the longer, straight chained acids, with an equivalent reduction in acetate acceptance [7]. There are other examples of this approach leading to acyl-CoA synthetases with substantially increased substrate scopes and although none have so far been paired with an NAT for the synthesis of amides, they do show the potential of utilising structural information to engineer a more substrate promiscuous CoA-ligase [35, 36]. As an alternative to engineering programs, high throughput screening has also been used to identify CoAligases and secondary $N$-acyltransferases (NATs) with complementary substrate profiles that can be combined to couple generated CoA-activated fatty acids to amine substrates for the generation of desired amine products [5].

The PCAS BioW has been shown to exhibit proof reading activities (hydrolysing the "wrong" acyl-adenylate) that limit its utility in producing novel structures (the enzyme is at least 100 times faster with pimelic acid than with either suberic acid or azelaic acid) [12]. However recent crystallography studies with pimelic acid, ATP and COA bound, as well as adenylate bound structures, have identified four key residues ( $B$. subtilis BioW Y199, T211, R213 and R227) responsible for the exquisite recognition of the di-acid substrate. Structure guided mutagenesis produced a couple of variants of the enzyme (Y199F and Y211F) that altered the substrate scope of the enzyme. Y199F retained activity with pimelic acid but was also able to convert heptanoic, 7octenoic, 7-phenylheptanoic, 6-methylheptanoic, and 7-bromoheptanoic acid to the corresponding CoA-conjugates [13]. This highlights that members of this new structural class of the ANL family may also have potential as biocatalysts for production of fatty acid CoA products. Members of the class I sub-family of the adenylate forming ligase family (Figure 1B) have seen most synthetic utility as 
ABS biocatalysts, but further engineering of classes II-IV may expand their substrate scope to accept amines and enable the preparation of useful amide containing products.

Finally, ATP-grasp enzymes have also been engineered to create new products. The D-Ala-DAla synthetase from Thermus thermophiles (TtDdL) was modified to produce amino acid amides from amino acids by mutating the active site residue responsible for binding the second alanine substrate [37]. One of these mutants (S293E) showed good conversion of D-alanine to the corresponding amine, however while the mutant also converted D-aminobutyric acid and glycine, no other amino acids could be converted and the enzyme was selective for D-amino acids [37].

\section{Conclusions and Prospects}

The synthesis of amide bonds is of very high value and traditional chemistries, while effective, have many drawbacks. Nature has an abundance of ATP-dependent amide forming enzymes which function, by different mechanisms, on a diverse range of substrates. The relatively simple amide bond synthetases (ABS) and ATP-grasp enzymes are most appealing for synthetic applications in vitro, as they function as standalone enzymes that can catalyse the direct formation of an amide. The combination of acyl-CoA synthetase (ACS) and $\mathrm{N}$-acyltransferase (NAT) enzymes has also been explored as a route to amides. However, such systems require ACS-NAT to have matched selectivity, which for more ambitious amide targets probably necessitates engineering of both enzymes. Complex multienzymes, such as NRPS, whilst providing valuable bioactive natural products are difficult to engineer as biocatalysts for simple amide synthesis. Adenylation domains, isolated from such multienzymes, can catalyse activation of carboxylic acids substrate as acyl-AMPs for nonenzymatic coupling with amines. However, acyl-AMP are unstable, with background hydrolysis effectively depleting valuable ATP, and their coupling with amines is slow which requires a large excess (100 eq.) of amine in some cases. Whilst a few amide forming enzymes, have been shown to exhibit some substrate promiscuity, the diversity of non-native amides that can be produced enzymatically is low. However there have been a few examples that demonstrate that protein engineering can be successful in developing more versatile ligases as future biocatalysts. Moreover, the fact that amides are ubiquitous in nature, including a large number of bioactive amidecontaining natural products indicates that there are many more amide bond forming enzymes that have yet to be discovered. Genome mining efforts will no doubt continue to identify promising new enzymes for characterisation and development in amide synthesis. Finally, the vast majority of amide forming enzymes require stoichiometric ATP, which is prohibitively expensive for use in vitro at scale. However, a wide range of ATP and CoA recycling methodologies have been developed, 
which could potentially enable amide bond forming enzymes to operate at scale [6, 38-41]. In addition, the prospect of using amide ligases in vivo under fermentation conditions is appealing, particularly if either the carboxylic acid or amine substrates can be generated metabolically from existing or engineered pathways.

\section{Acknowledgements}

Research in the JM and MW is supported by the BBSRC (grant BB/K002341/1) and Syngenta. Research of DJC and SR is supported by

\section{References}

[1] Dunetz JR, Magano J, Weisenburger GA: Large-scale applications of amide coupling reagents for the synthesis of pharmaceuticals. Org Process Res Dev. 2016, 20:140-177.

[2] Carey JS, Laffan D, Thomson C, Williams MT: Analysis of the reactions used for the preparation of drug candidate molecules. Org Biomol Chem. 2006, 4:2337-2347.

[3] Valeur E, Bradley M: Amide bond formation: beyond the myth of coupling reagents. Chem Soc Rev. 2009, 38:606-631.

[4] Funabashi M, Yang ZY, Nonaka K, Hosobuchi M, Fujita Y, Shibata T, Chi XL, Van Lanen SG: An ATP-independent strategy for amide bond formation in antibiotic biosynthesis. Nat Chem Biol. 2010, 6:581-586.

*[5] Philpott HK, Thomas PJ, Tew D, Fuerst DE, Lovelock SL: A versatile biosynthetic approach to amide bond formation. Green Chem. 2018, 20:3426-3431.

In this study, CoA ligases and $\mathrm{N}$-acyltransferases with desired substrate profiles were combined to form non-natural biocatalytic pathways to access structurally diverse amides in vitro or in vivo.

[6] Mordhorst S, Maurer A, Popadic D, Brech J, Andexer JN: A flexible polyphosphate-driven regeneration system for coenzyme A dependent catalysis. ChemCatChem. 2017, 9:4164-4168

[7] Sofeo N, Hart JH, Butler B, Oliver DJ, Yandeau-Nelson MD, Nikolau BJ: Altering the substrate specificity of acetyl-CoA synthetase by rational mutagenesis of the carboxylate binding pocket. ACS Synth Biol. 2019, 8:1325-1336.

[8] Goswami A, Van Laren SG: Enzymatic strategies and biocatalysis for amide bond formation: tricks of the trade outside of the ribosome. Mol. Biosyst. 2015, 11:338-353.

[9] Pitzer J, Steiner K: Amides in nature and biocatalysis. J. Biotechnol. 2016, 235:32-46

[10] Dorr BM, Fuerst DE: Enzymatic amidation for industrial applications. Curr. Opin. Chem. Biol. 2018, 43:127-133

[11] Petchey MR, Grogan G: Enzyme-catalysed synthesis of secondary and tertiary amides. Adv. Synth. Catal. 2019, 361:3895-3914 
[12] Estrada P, Manandhar M, Dong SH, Deveryshetty J, Agarwal V, Cronan JE, Nair SK: The pimeloyl-CoA synthetase BioW defines a new fold for adenylate-forming enzymes. Nat Chem Biol. 2017, 13:668-647.

[13] Wang ML, Moynie L, Harrison PJ, Kelly V, Piper A, Naismith JH, Campopiano DJ: Using the pimeloyl-CoA synthetase adenylation fold to synthesize fatty acid thioesters. Nat Chem Biol. 2017, 13:660-667.

[14] Steffensky M, Muhlenweg A, Wang ZX, Li SM, Heide L: Identification of the novobiocin biosynthetic gene cluster of Streptomyces spheroides NCIB 11891. Antimicrob Agents Chemothr. 2000, 44:1214-1222.

[15] Eustaquio AS, Gust B, Luft T, Li SM, Chater KF, Heide L: Clorobiocin biosynthesis in Streptomyces: Identification of the halogenase and generation of structural analogs. Chemistry \& Biology. 2003, 10:279-288.

[16] Yang Y, Fu L, Zhang JL, Hu LH, Xu MJ, Xu J: Characterization of the xiamenmycin biosynthesis gene cluster in Streptomyces xiamenensis 318. Plos One. 2014, 9:e99537.

[17] Galm U, Dessoy MA, Schmidt J, Wessjohann LA, Heide L: In vitro and in vivo production of new aminocoumarins by a combined biochemical, genetic, and synthetic approach. Chemistry \& Biology. 2004, 11:173-183.

*[18] Ji CT, Chen Q, Li QL, Huang HB, Song YX, Ma JY, Ju JH: Chemoenzymatic synthesis of betacarboline derivatives using McbA, a new ATP-dependent amide synthetase. Tetrahedron Lett. 2014, 55:4901-4904.

Here the ATP-dependent amide synthetase McbA was characterised and utilised to form amide bonds with a broad range of substituted amides to form 10 new $\beta$-carboline analogues.

**[19] Petchey M, Cuetos A, Rowlinson B, Dannevald S, Frese A, Sutton PW, Lovelock S, Lloyd RC, Fairlamb IJS, Grogan G: The broad aryl acid specificity of the amide bond synthetase McbA suggests potential for the biocatalytic synthesis of amides. Angew Chem Int Ed. 2018, 57:1158411588.

In this study the substrate specificity of McbA was further explored and a range of pharmaceuticaltype amides were enzymatically generated from a range of aryl carboxylic acids

[20] Arai T, Arimura Y, Ishikura S, Kino K: L-amino acid ligase from Pseudomonas syringae producing tabtoxin can Be used for enzymatic synthesis of various functional peptides. Appl Environ Microb. 2013, 79:5023-5029.

[21] Isono K, Suhadolnik RJ: Biosynthesis of natural and unnatural polyoxins by Streptomyces cacaoi. Arch Biochem Biophys. 1976, 173:141-153.

[22] Gong R, Qi JZ, Wu P, Cai YS, Ma HM, Liu Y, Duan H, Wang M, Deng ZX, Price NPJ, Chen WQ: An ATP-dependent ligase with substrate flexibility involved in assembly of the peptidyl nucleoside antibiotic polyoxin. Appl Environ Microb. 2018, 84.

**[23] Noike M, Matsui T, Ooya K, Sasaki I, Ohtaki S, Hamano Y, Maruyama C, Ishikawa J, Satoh Y, Ito $\mathrm{H}$, Morita $\mathrm{H}$, Dairi T: A peptide ligase and the ribosome cooperate to synthesize the peptide pheganomycin. Nat Chem Biol. 2015, 11:71-76. 
In this study PGM1, an ATP-grasp enzyme, is described and characterised. PGM1 was found to accept ribosomally synthesised peptides as substrates for ligation with non-proteinogenic amino acids. Structural studies highlighted a large peptide binding pocket that helps to explain the broad range of accepted peptides.

[24] Ooya K, Ogasawara Y, Noike M, Dairi T A peptide ligase and the ribosome cooperate to synthesize the peptide pheganomycin. Biosci Biotech Bioch. 2015, 79:1833-1837.

[25] Ogasawara Y, Kawata J, Noike M, Satoh Y, Furihata K, Dairi T: Exploring peptide ligase orthologs in actinobacteria-discovery of pseudopeptide natural products, ketomemicins. ACS Chem Biol. 2016, 11:1686-1692.

[26] Winn M, Fyans JK, Zhuo Y, Micklefield J: Recent advances in engineering nonribosomal peptide assembly lines. Nat Prod Rep. 2016, 33:317-347.

[27] Kries $\mathrm{H}$, Niquille DL, Hilvert D: A subdomain swap strategy for reengineering nonribosomal peptides. Chemistry \& Biology. 2015, 22:640-648.

* [28] Hara R, Hirai K, Suzuki S, Kino K: A chemoenzymatic process for amide bond formation by an adenylating enzyme-mediated mechanism. Sci Rep. 2018, 8:2950.

In this study the first adenylation domain of tyrocidine synthetase 1 was isolated and combined with primary or secondary amines, as nucleophiles, to synthesise a range of tryptophyl- $N$ alkylamides.

[29] Zhu M, Wang L, He J: Chemical diversification based on substrate promiscuity of a standalone adenylation domain in a reconstituted NRPS system. ACS Chem Biol. 2019, 14:256265.

[30] Marchetti PM, Kelly V, Simpson JP, Ward M, Campopiano DJ: The carbon chain-selective adenylation enzyme TamA: the missing link between fatty acid and pyrrole natural product biosynthesis. Org Biomol Chem. 2018, 16:2735-2740.

*[31] Marchetti PM, Richardson SM, Kariem NM, Campopiano DJ: Synthesis of $\mathbf{N}$-acyl amide natural products using a versatile adenylating biocatalyst. Med. Chem. Commun. 2019, 10:11921196.

Here the isolated adenylation domain from enzyme TamA is utilised to generate a range of acyl adenylates that can be captured by a number of amines to generate a series of fatty $\mathrm{N}$-acyl amides including members of $\mathrm{N}$-acyl histidine amide class of natural products.

[32] Gahloth D, Dunstan MS, Quaglia D, Klumbys E, Lockhart-Cairns MP, Hill AM, Derrington SR, Scrutton NS, Turner NJ, Leys D: Structures of carboxylic acid reductase reveal domain dynamics underlying catalysis. Nat Chem Biol. 2017, 13:975-981.

[33] Winkler M: Carboxylic acid reductase enzymes (CARs). Curr Opin Chem Biol. 2018, 43:23-29.

*[34] Wood AJL, Weise NJ, Frampton JD, Dunstan MS, Hollas MA, Derrington SR, Lloyd RC, Quaglia $D$, Parmeggiani F, Leys D, Turner NJ, Flitsch SL: Adenylation activity of carboxylic acid reductases enables the synthesis of amides. Angew Chem Int Ed. 2017, 56:14498-14501. 
In this study carboxylic acid reductases (CARs) were utilised to form amide bonds between a range of carboxylic acids and amines. Optimisation of the reaction conditions allowed the generation of the anticonvulsant ilepcimide with up to $96 \%$ conversion.

[35] Ingram-Smith C, Woods BI, Smith KS: Characterisation of the acyl substrate binding pocket of acetyl-CoA synthetase. Biochemistry. 2006, 38:11482-11490.

[36] Thornburg CK, Wortas-Strom S, Nosrati M, Geiger JH, Walker KD: Kinetically and crystallographically guided mutations of a benzoate CoA ligase (BadA) elucidate mechanism and expand substrate permissivity. Biochemistry. 54:6230-6242.

[37] Miki Y, Okazaki S, Asano Y: Engineering an ATP-dependent D-Ala:D-Ala ligase for synthesizing amino acid amides from amino acids. J Ind Microbiol Biot. 2017, 44:667-675.

[38] Resnick SM, Zehnder AJB: In vitro ATP regeneration from polyphosphate and AMP by polyphosphate: AMP phosphotransferase and adenylate kinase from Acinetobacter johnsonii 210A. Appl Environ Microb. 2000, 66:2045-2051.

[39] Andexer JN, Richter M: Emerging enzymes for ATP regeneration in biocatalytic processes. Chembiochem. 2015, 16:380-386.

[40] Alissandratos A, Caron K, Loan T. D, Hennessy JE, Easton CJ: ATP recycling with cell lysate for enzyme-catalyzed chemical synthesis, protein expression and PCR. ACS Chemical Biology. 2016, 11:3289-3293.

[41] Strohmeier GA, Eiteljorg IC, Schwarz A, Winkler M: Enzymatic one-step reduction of carboxylates to aldehydes with cell-free regeneration of ATP and NADPH. CHEM-EUR J. 2019, 25:6119-6123. 
A

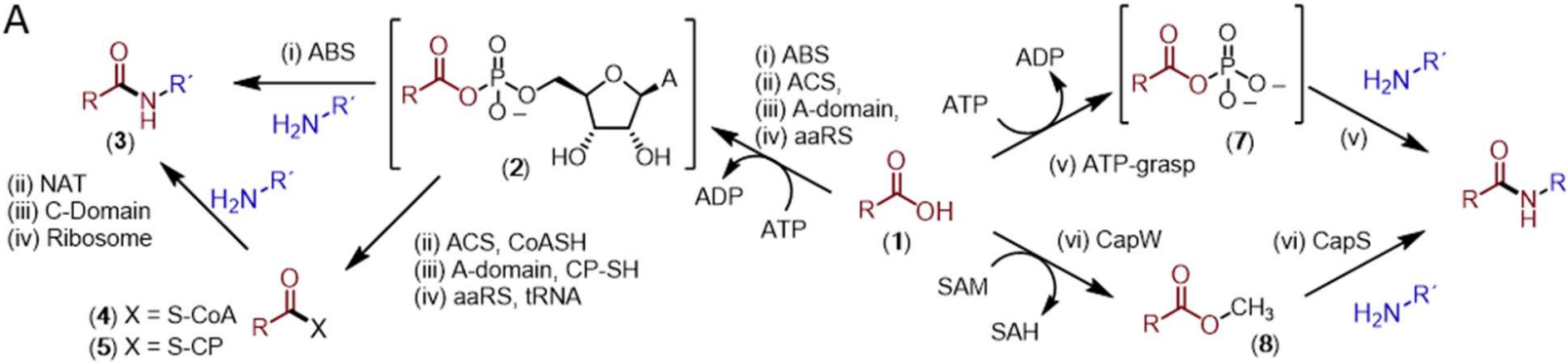

(6) $X=$ O-tRNA

B

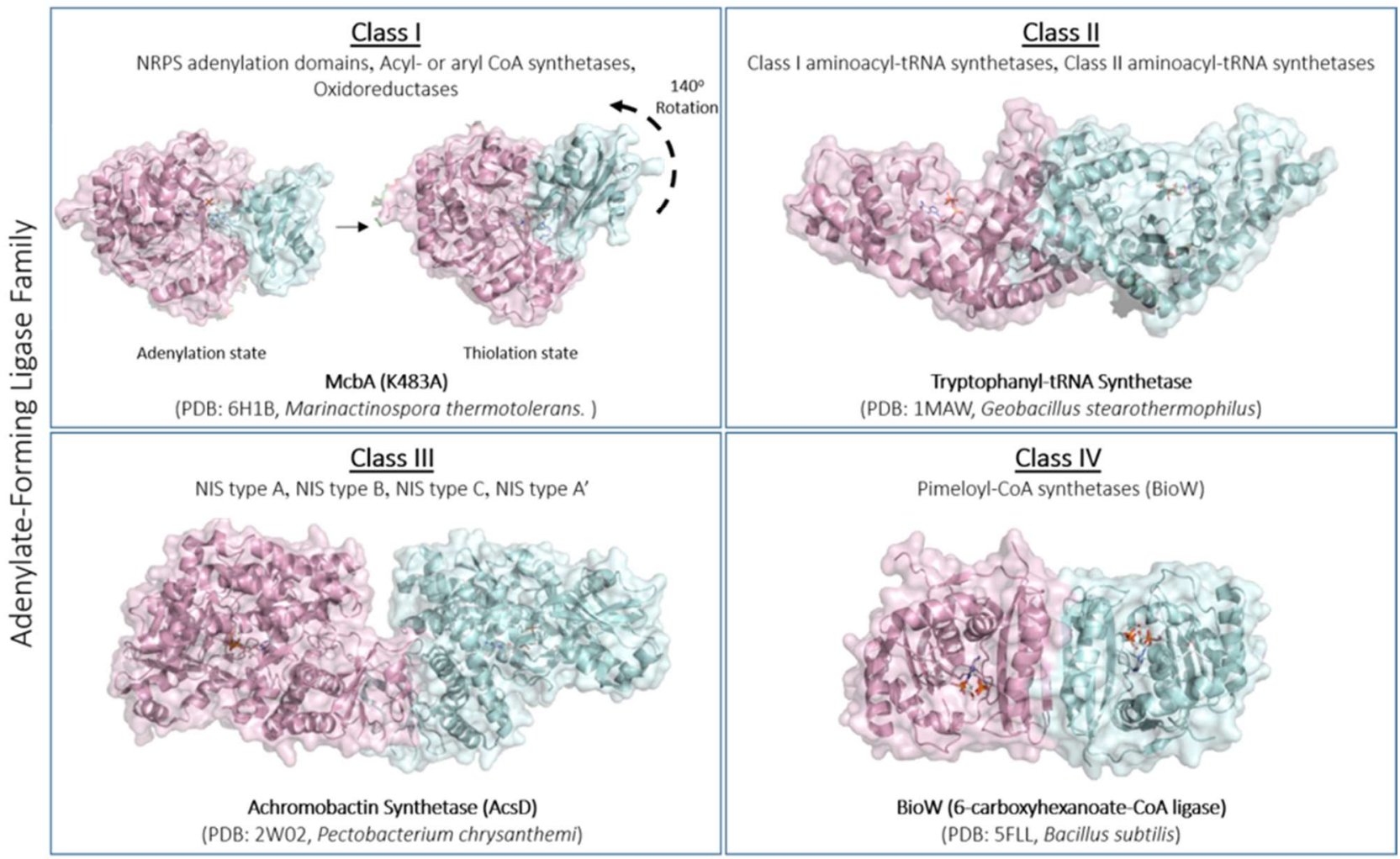

Figure 1. Biosynthesis of amides. (A) The majority of enzymatic routes to amides involve activation of a carboxylic acid substrate (1) by ATP to generate an acyl adenylate (acyl-AMP) (2) including: (i) amide bond synthetase (ABS) where the acyl-AMP (2), is attacked directly by an amine to generate the amide (3); (ii) acyl-CoA synthetase (ACS) produce intermediate acyl-CoA thioesters (4), which can be coupled with amine in a reaction catalysed by a second $N$-acyltransferase (NAT) enzyme; (iii) in nonribosomal peptide synthetase (NRPS) enzymes an adenylation (A) domain generates an amino acyl-AMP (2) leading to a carrier protein (CP) thioester intermediate (5) a distinct condensation (C) domain then catalyses peptide bond formation; (iv) aminoacyl-tRNA synthetase (aaRS) enzymes produce amino acyl-tRNAs (oxy-esters, 6) which enable ribosomal peptide bond formation. Alternatively, ( $v$ ) the ATP-grasp enzymes, catalyse formation of acyl phosphates (7) and subsequent amide formation. (vi) CapW and CapS, provide a rare example of an ATP-independent amide biosynthesis, were CapW SAM-dependant methylation gives a methyl ester (8) that is subsequently coupled with an amine by CapS. (B) Representative structures of adenylate-forming enzymes: Class I McbA PDB Code: 6H1B (C-terminus (blue) and N-terminus (Pink) with AMP and 1-acetyl-3-carboxy- $\beta$-carboline bound); Class II Trp Synthetase PDB Code: 1MAW (Open conformation with one monomer in pink and the other in blue with ATP bound ); Class III Achromobactin Synthetase PDB Code: 2W02 (dimer with one monomer in pink and the other in blue with ATP bound); Class IV BioW PDB Code: 5FLL (dimer with one monomer in pink and the other in blue with pimeloyladenylate bound). 
A

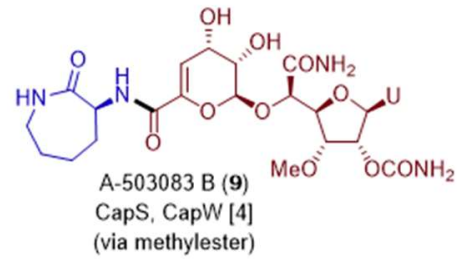

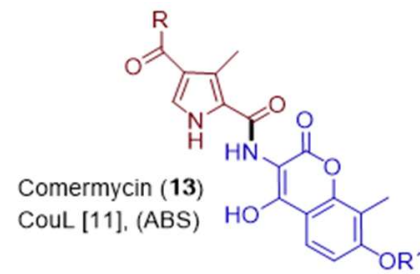

$\underset{\substack{\text { Polyoxin J (15) } \\ \text { Polg [15], (ATP-grasp) }}}{O C H}$

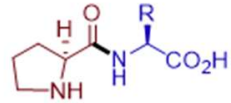

Tabs [14] (ATP-grasp

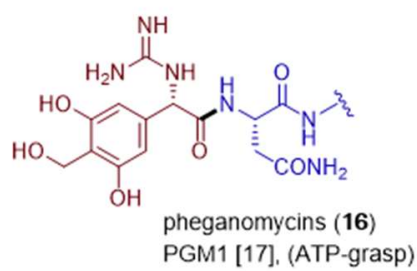
PGM1 [17], (ATP-grasp)

Xiamenmycin (12)<smiles>CC(C)=CCC[C@]1(C)Oc2ccc(C(=O)N[C@H](C(=O)O)C(C)O)cc2C[C@H]1O</smiles>
NovL [11]: $\mathrm{R}=\mathrm{Cl}$, Clorobiocin (9), $\mathrm{CloL}[11]$
(ABS) XimA [10], (ASB)

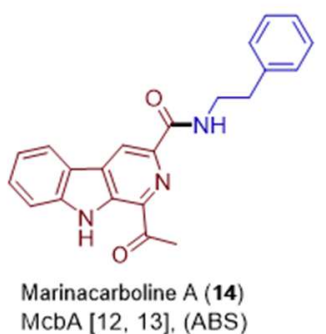

B<smiles>[R]c1nc(C(=O)O)cc2c1[nH]c1ccccc12</smiles><smiles>O=C(O)c1ccccc1</smiles><smiles>O=C(O)c1ccc2ccccc2c1</smiles><smiles>NC1CCCC1</smiles><smiles>N=C(N)c1ccccc1</smiles><smiles>CC(N)c1ccccc1</smiles><smiles>CC(N)CCc1ccccc1</smiles><smiles>[R]=CC(C)C(C)O</smiles><smiles>O=C(O)c1c[nH]c2ccccc12</smiles><smiles>O=C(O)c1ccc2[nH]ccc2c1</smiles><smiles>O=C(O)c1ccc2occc2c1</smiles><smiles>CC(=O)c1cccc(C(=O)O)n1</smiles><smiles>O=C(O)c1cc2ccccc2cn1</smiles><smiles>[R]c1ccccc1CCN</smiles>

$\mathrm{R}=\mathrm{OH}, \mathrm{OCH}_{3}, \mathrm{Br}, \mathrm{CH}_{3}$<smiles>[R]c1cccc(CCN)c1</smiles><smiles>NCCc1cc2ccccc2[nH]1</smiles><smiles>COc1ccc2[nH]c(CCN)cc2c1</smiles>

Figure 2 (A) Examples of secondary metabolites produced by ABS and ATP-grasp enzymes, as well as the unusual A-503083 (9) natural product generated by CapS and CapW via an intermediate methylester [4]. (B) Substrate scope of McbA with donor acid substrates in red and amines in blue $[18,19]$. 
A
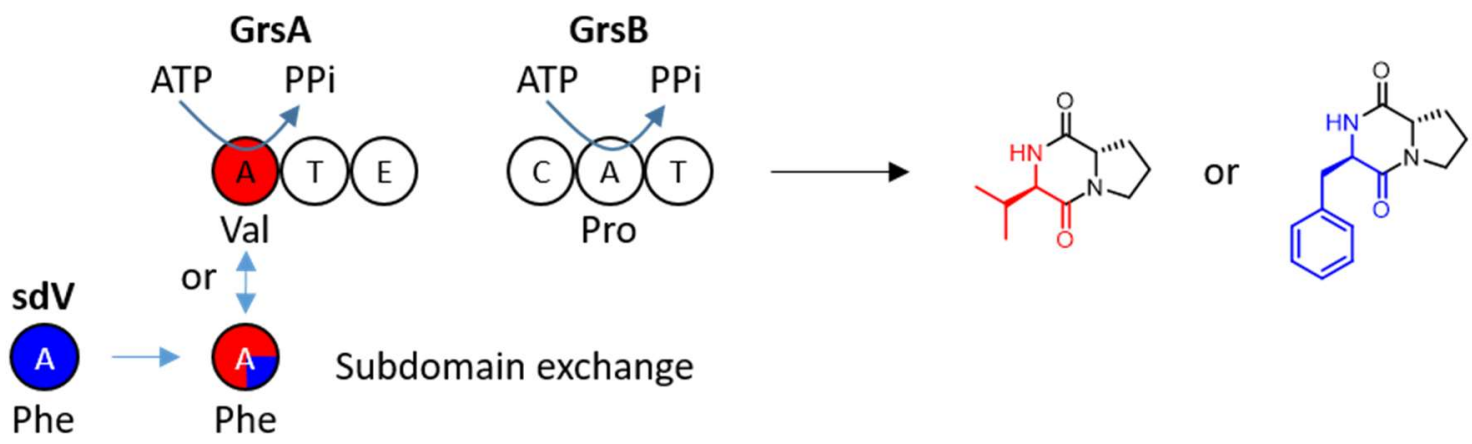

B

SfaB

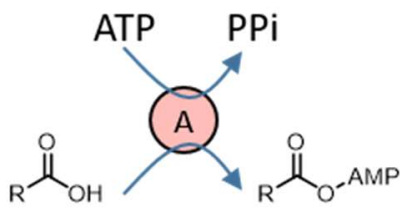

C

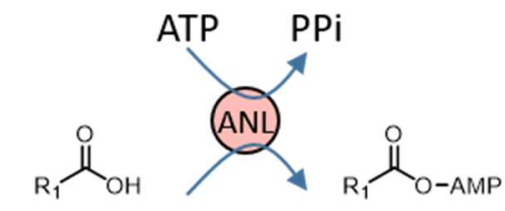

$\mathrm{C}_{6}-\mathrm{C}_{12}$ fattly acids

TamA

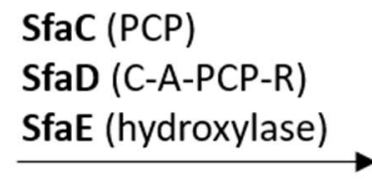<smiles>[R]C(=O)NCC1CCC(NC([R])=O)OC1O</smiles>

SF2768 (17) analogs<smiles>C=CC(C)C(CC)C(=C)CC</smiles>

D

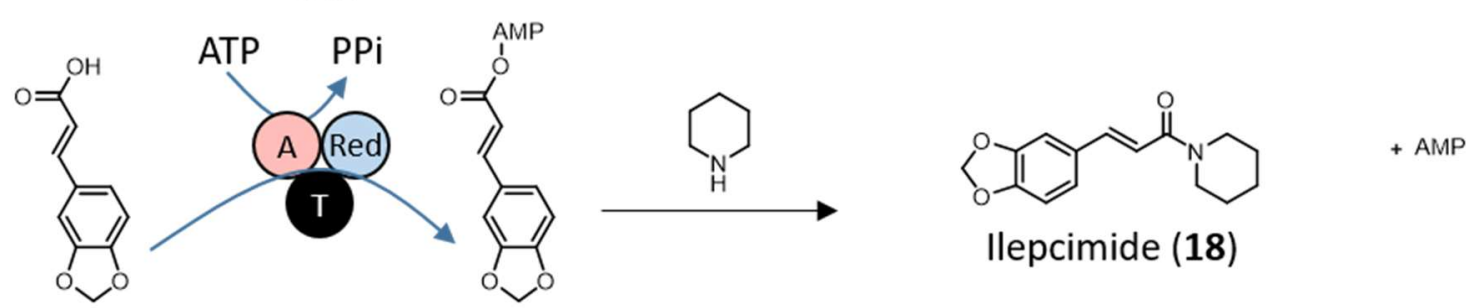

Figure 3. Examples of engineered amide ligase systems for the preparation of useful amide bond containing products. (A) Subdomains within the Phe activating adenylation (A) domain of NRPS protein GrsA can be exchanged with a subdomain from alternative Val activating $A$ domain from GrsB to make a modified peptide product. Preferred substrate of each A domain is indicated below the circle [27]. (B) Producing the adenylation domain SfaB in isolation afforded a catalyst that could accept a range of fatty acids. Combining this with a number of other genes from the parent pathway allowed the generation of a number of novel SF2768 analogs [29]. (C) An ANL found within the tambjamine biosynthetic pathway produced in isolation can generate a range of fatty $\mathrm{N}$-acyl amines [31]. (D) A carboxylic acid reductase (CAR) has been repurposed for the generation of ilepcimide (18) when the reductase domain is inactivated by feeding of amines in the absence of NADH [34]. T - thiolation/PCP domain, E - epimerisation domain, C - condensation domain, Red - reductase domain. 